\title{
Mobile Radio Evolution
}

\author{
M. Prasad, R. Manoharan \\ Dept. of Computer Science and Engineering, Pondicherry Engineering College, Puducherry, India
}

Email address:

prasad.psd@gmail.com (M. Prasad), rmanoharan@yahoo.co.in (D. R. Manoharan)

\section{To cite this article:}

M. Prasad, Dr. R. Manoharan. Mobile Radio Evolution. Advances in Networks. Special Issue: Secure Networks and Communications. Vol. 3, No. 3-1, 2015, pp. 1-6. doi: 10.11648/j.net.s.2015030301.11

\begin{abstract}
All over the world, wireless communication services have enjoyed dramatic growth over the past 25 years. Mobile communication is the booming field in the telecommunications industry. The cellular network is the most successful mobile communication system, used to transmit both voice and data. This paper provides a depth view about the technologies in mobile communication from the evolution of the mobile system. First from the evolution, second generation $(2 \mathrm{G})$, third generation $(3 \mathrm{G})$, fourth generation $(4 \mathrm{G})$ to fifth generation $(5 \mathrm{G})$ in terms of performance requirements and characteristic.
\end{abstract}

Keywords: 2G, 3G, 4G, 5G, AMPS, GPRS, UMTS, HSDPA

\section{Introduction}

The Detroit Police Department radio bureau began experimentation in 1921 with a band near $2 \mathrm{MHz}$ for vehicular mobile service. On April 7, 1928 the Department started regular one way radio communication with patrol cars to communicate a central control point. It established the practicality of land mobile radio for police work and lead to it does adopt throughout the country. Channels in this frequency band soon became crowded. Figure 1 shows the evolution of mobile radio.

In 1933 the police department in Bayonne, New Jersey started regular two way communications with patrol cars [1]. The very high frequency transmitters are placed in patrol cars to enable patrolmen to communicate with headquarters and other cars instead of just receiving calls. Two way communication of police radio became standard throughout the country.

World War II proved [2] that the production of VHF radios was possible, by the end of the 1940's the development of mobile communications systems are initiated.

In 1940 new frequencies allocated between $30-40 \mathrm{MHz}$ leads to cover a major distance with police radio systems. Police radio major evolution occurred when the Connecticut State Police started operating a two way frequency modulated (FM) [3] system in Hartford is the breakthrough in mobile radio. The state wide two way radio system was developed by Daniel E. Noble of the University of Connecticut and engineers at the Fred M. Line Company greatly reduced the main problem of the amplitude modulated (AM) system. FM mobile radio became standard all over the country. Federal Communications Commission (FCC) allocates $40 \mathrm{MHz}$ of spectrum in range between 30 and $500 \mathrm{MHz}$ for private individuals, companies, and public agencies for mobile services.

Late 1940's Bell System gets into a program of supplying "public correspondence systems". FCC classified public correspondence systems services as Domestic Public Land Mobile Radio Service (DPLMRS) [4]. This allows communication among public and private sectors provided by a common carrier.

By 1946 First Bell, "urban” DPLMRS was inaugurated in St. Louis, it became possible to link a vehicle mounted mobile radio unit to a Public Switched Telephone Network (PSTN) with three channels near $150 \mathrm{MHz}$, manually patched.

By 1947 A 35 to $44 \mathrm{MHz}$ "highway" system between New York and Boston was inaugurated. It was thought that these frequencies would carry further along highways.

By 1950 Private Mobile Radio \& Private Access Mobile Radio (PMR \& PAMR) systems were developed. These are usually utilising vehicle mounted units which are mainly used by the emergency services, public utilities, road haulage and taxis. These systems were very basic single site and single channel, they have developed into much more complex systems, utilising multisite, multichannel, selective calling, connections PABXs/PSTN's and direct calling Terrestrial Trunked Radio (TETRA) [5].

All of the above mentioned services employed by push-to-talk (PTT) operation. Radio is half duplex which is unfamiliar and awkward for ordinary phone users and it 
required an operator to place a call [6]. Most of these services were not directly part of the PSTN but it involved a radio channel which was patched into a phone line.

By 1964 First automatic $150 \mathrm{MHz}$ service (called MJ) was introduced, for this free channel is automatically assigned and the system was full duplex and customers could do their own dialing. By 1969 First automatic $450 \mathrm{MHz}$ service (called MK) was introduced and extended MJ to a new band. Combining these two services became the Improved Mobile Telephone Service (ITMS) the standard until the development of AMPS. Table 1 gives the details about the technologies and their features.

Table 1. Overview of Technologies.

\begin{tabular}{llll}
\hline & Technology & Speed & Features \\
\hline $1 \mathrm{G}$ & AMPS & n/a & Analog \\
$2 \mathrm{G}$ & GSM, CDMA, iDen & Less than 20Kbps & Voice, SMS, Conference calls \\
$2.5 \mathrm{G}$ & GPRS, EDGE & 30Kbps to 90Kbps & MMS, image, web browsing, games applications \\
$3 \mathrm{G}$ & UMTS, 1xEV-DO & $144 \mathrm{Kbps}$ to $2 \mathrm{Mbps}$ & Full motion video music streaming and faster web. \\
$3.5 \mathrm{G}$ & HSDPA & $384 \mathrm{Kbps}$ to 14.4 Mbps & On Demand video, video conferencing \\
$4 \mathrm{G}$ & WiMax, LTE-A & 100Mbps to 1 Gbps & High quality streaming video, voice over IP telephony, VoLTE service. \\
$5 \mathrm{G}$ & WWWW & Beyond 1 Gbps & Internet \\
\hline
\end{tabular}

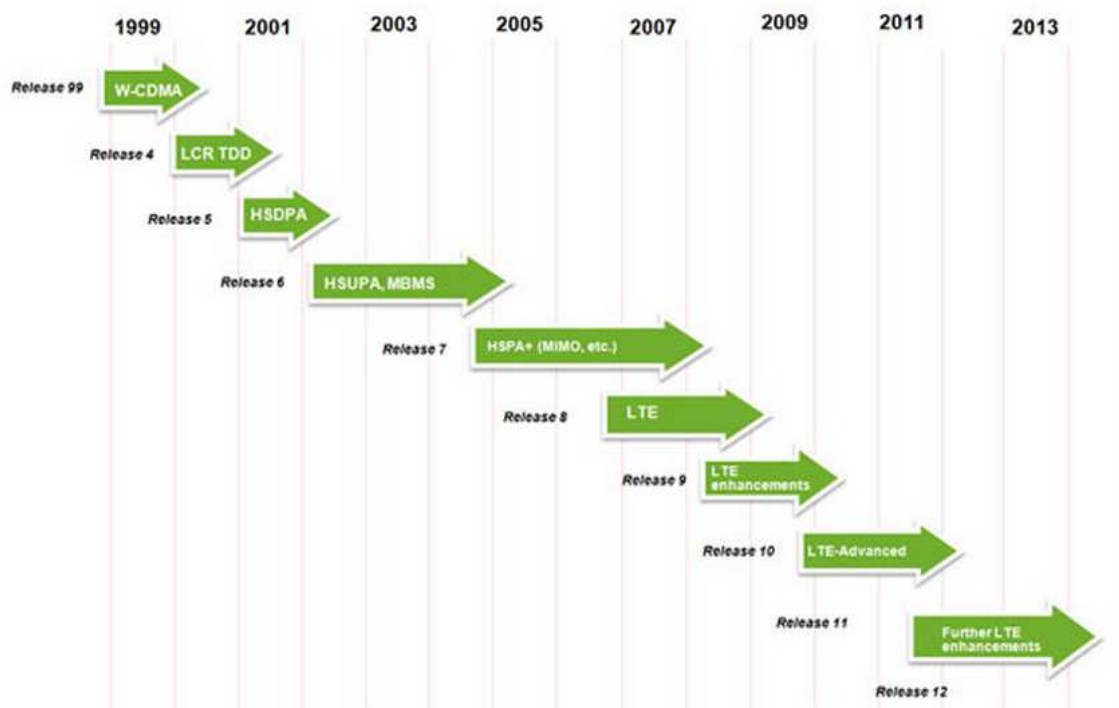

Figure 1. Radio Access (source 3GPP.org).

FCC debates frequency allocation to common carriers. By 1974 it approved the underlying concepts of wireless cellular phone service and allocated for this purpose 666 duplex (two way) channels in the $800-900 \mathrm{MHz}$ frequency range and the authorization granted to Illinois Bell in 1978 [7]. Advanced Mobile Phone Service (AMPS) trials begin (850 MHz) in Chicago and American Radio Telephone Service (ARTS) in Washington DC. In 1981 Nordic Mobile Telephone System (NMT) enters public service in Sweden developed by Ericsson with frequencies in the 450 to 470 band. World Administrative Radio Conference sets up research group to define a common international standard it leads to Group Speciale Mobile (GSM) in 1982.

\section{Mobile Radio Telephone}

In 1984 AMPS cellular system was initially deployed. It is an analog mobile phone system standard developed by Bell Labs. It was the first generation cellular technology that uses separate frequencies, or "channels", for each conversation Frequency Division Multiple Access (FDMA) was used. It therefore required considerable bandwidth for a large number of users. AMPS was very similar to the older " $0 \mathrm{G}$ ". Improved
Mobile Telephone Service used considerably more computing power in order to select frequency, hand off conversations to PSTN lines and call setup.

Since it was an analog standard, it is very familiar to static and noise and has no protection from eavesdropping. In the 1990s, the industry loses millions of dollars due to cloning of handset. An eavesdropper with specialized equipment could intercept a handset's ESN (Electronic Serial Number) and MIN (Mobile Identification Number, aka the telephone number). It is easy to clone the handset when the ESN/MIN pair is identified and the clone is used in other areas for making calls without paying.

AMPS was originally standardized by ANSI as EIA/TIA/IS-3. EIA/TIA/IS-3 was replaced by EIA/TIA-553 and TIA interim standard IS-91 (Narrowband Advanced Mobile Phone System). Very soon AMPS networks have been replaced by digital networks based on standards including Digital AMPS, GSM, and CDMA2000 which brought with improved security as well as increased bandwidth. Cloning is still possible even with digital technologies, the cost of wireless service is reduced so that the problem has virtually disappeared. 


\section{Second Generation}

Second generation $2 \mathrm{G}$ cellular telecom networks were commercially launched on the Global System for Mobile communication (GSM) standard in Finland by Radiolinja in 1991. The primary benefits of $2 \mathrm{G}$ networks over their predecessors were that phone conversations were digitally encrypted. $2 \mathrm{G}$ systems were significantly more efficient on the spectrum allowing for far greater mobile phone penetration levels. $2 \mathrm{G}$ system introduced data services for mobile, starting with SMS text messages. Depending on the type of multiplexing $2 \mathrm{G}$ technologies can be divided into TDMA-based and CDMA-based standards. The $2 \mathrm{G}$ version of Code Division Multiple Access (CDMA) is referred to as cdmaOne. These digital systems evolved to form the second generation or $2 \mathrm{G}$. In the late 1980 GSM was developed by the European Telecommunications Standard Institute (ETSI), and in 1993 the IS-95 CDMA standard was introduced by the TR45.5 subcommittee of the Telecommunications Industry Association (TIA).

The GSM system is a TDMA radio system with carrier bands that are $200 \mathrm{KHz}$ wide. Each band is comprised of eight bearer slot and it was a circuit switched system.

CdmaOne uses a different radio technology referred to as "spread spectrum" where the radio spectrum is divided into carriers which is approximately 1.23 MHz's wide. Each voice channel is assigned a unique code within the carrier and the voice signal is spread to a transmitted rate of about 1.23 Megabits per second. The cdmaOne [10] network utilizes universal frequency reuse where the same frequency can be reused in every cell. This enables greater capacity as compared to the TDMA based systems.

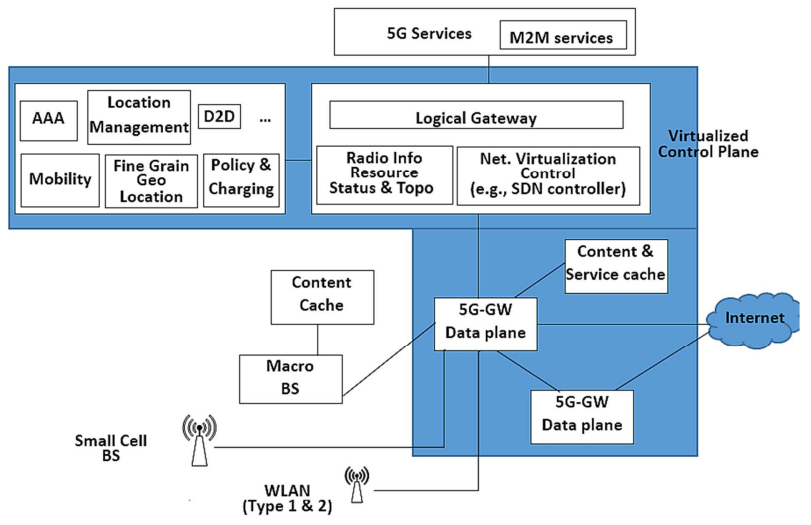

Figure 2. $5 G$ Architecture.

The $2 \mathrm{G}$ system support basic data services with limited capacity since the single voice channel is used for the data transmission. Only one wireless bearer slot of the GSM carrier band is allocated to the data transfer so that the transfer rate is limited to $9.6 \mathrm{kbps}$. An improvement of this scheme was made available in the form of high speed circuit switched data (HSCSD) where multiple bearer slots are made available to the same call.

ETSI developed the general packet radio service (GPRS), a packet transmission system that incorporate with GSM and interworks with external packet data networks such as internet. $2.5 \mathrm{G}$ wireless communication system is called as GPRS. An IP address is assigned to each mobile terminal in GPRS system. The assignment may be static or dynamic depends on the cellular operator on a per connection basis. A GPRS enabled mobile terminal can use between one to eight wireless bearer slots of the GSM carrier band. The higher the number of assigned slots makes faster data transfer with speeds of up to $115 \mathrm{kbps}$.

The GSM network components used for handling cellular telephony calls are the BSS, MSC/VLR, and HLR.

The Base Station Subsystem (BSS) includes the Base Transceiver Station (BTS) and Base Station Controller (BSC). The BTS include antennas and handles the radio transmission to the mobile terminals. The BSC which manages several BTSs. The BSC transmits voice calls to the Mobile Switching Center (MSC) and contains the Packet Control Unit (PCU) for handling data traffic to the GPRS network.

The MSC switches voice calls between the mobile terminals and the Public Switch Telephone Network (PSTN). MSC handles the setup calls and allocation of circuit between mobile terminals or between mobile terminal and PSTN. The Visitor Location Register (VLR) often collocates with the MSC. This database stores the temporary information's about the mobile terminal in the cell site.

The Home Location Register (HLR) database contains the mobile subscribers profile information that includes the list of subscribed services. The mobile terminals are authenticated by HLR that want to access the mobile network and also records the mobile terminal locations in the network.

The GPRS packet network includes two new supporting nodes, the Serving GPRS Support Node (SGSN) and the Gateway GPRS Support Node (GGSN). The traffic from mobile terminal is split at the BSC with voice sent to the MSC and data packets sent to the SGSN. The GPRS mobile terminals are tracked by the SGSN in the cell site and for routing data packets to the mobile terminals. The GGSN serves as a router that interfaces between the internet, or other packet data network, and the IP-based GPRS network. The GGSN allocates IP address for the mobiles in the cell site and routes mobile destined packets to the appropriate SGSN. GPRS can be used for various services such as Wireless Application Protocol (WAP) access, Multimedia Messaging Service (MMS), and for Internet communication services such as email and World Wide Web access.

GPRS networks evolved to EDGE networks by 8PSK encoding introduction. Enhanced Data rates for GSM Evolution (EDGE), Enhanced GPRS (EGPRS), or IMT Single Carrier (IMT-SC) is a backward-compatible digital mobile phone technology that allows improved data transmission rates. EDGE was deployed on GSM networks beginning in 2003.

EDGE is standardized by 3rd Generation Partnership Project (3GPP) as part of the GSM family and it is an upgrade that provides a potential three-fold increase in capacity of GSM/GPRS networks. The specification achieves higher data rates up to $236.8 \mathrm{kbit} / \mathrm{s}$ by switching to more sophisticated 
methods of coding (8PSK), within existing GSM timeslots [8].

\section{Third Generation}

The increasing in the demand for data services began to work on the next generation of technology known as $3 \mathrm{G}$ third generation. The main technological difference in the $3 \mathrm{G}$ and $2 \mathrm{G}$ networks is the use of packet switching rather than circuit switching for data transmission with greater speed. The $3 \mathrm{G}$ standard for mobile communication services fulfils the International Mobile Telecommunications-2000 (IMT-2000) specifications by the International Telecommunication Union (ITU) [9]. Some of the standards are based on the wideband CDMA (W-CDMA), also referred to as Universal Mobile Telecommunications System (UMTS) these are worked by Third Generation Partnership Project (3GPP) established in 1998.

The data rate for transferring data between the mobile terminal and the network increase with each new cellular generation. The $3 \mathrm{G}$ systems are expected to deliver high data rates in the hundreds of kbps and up to 2 Mbps. UMTS employs a $5 \mathrm{MHz}$ channel carrier width to deliver these higher data rates and increased capacity. The main feature included in the design of $3 \mathrm{G}$ networks is the assurance of quality-of-service.

HSPA is upgrades from the original W-CDMA standard and offers speeds of 14.4Mbit/s download and 5.76MBit/s upload. HSPA is backwards compatible with $2 \mathrm{G}$ and uses the same frequencies as W-CDMA [11].

HSPA + is further revision and upgrade of HSPA, can provide theoretical peak data rates up to $168 \mathrm{Mbit} / \mathrm{s}$ in the downlink and $22 \mathrm{Mbit} / \mathrm{s}$ in the uplink, using a combination of air interface improvements as well as multi-carrier HSPA and MIMO.

IMT-2000 is the term used by the International Telecommunications Union for this set of globally harmonized standards. This initiative was to define the goal of accessing the global telecommunication infrastructure through both satellite and terrestrial mobile systems. IMT-2000 has revealed the explosion of mobile usage and the need for future high-speed data communications, with wideband mobile usage. IMT-2000 is a flexible standard that allows operators around the world the freedom of radio access methods and of core networks so that they can openly implement and evolve their systems.

Wideband Code Division Multiple Access (WCDMA) is completely different protocols should not be confused with narrowband CDMA. WCDMA is a latest technology, defined specifically to deliver high-speed data services and Internet-based packet-data at $3 \mathrm{G}$ data rates. WCDMA supports both packet and circuit-switched communications, such as Internet access and landline telephone services. WCDMA was defined with no requirements on second-generation backward compatibility. WCDMA makes very efficient use of the available radio spectrum. Frequency planning is not needed, since one cell re-use is applied. The techniques such as adaptive antenna arrays, hierarchical cell structures, and coherent demodulation, network capacity can be increased. Circuit and packet-switched services can be combined on the same channel, which allowing true multimedia services with multiple packet or circuit connections on a single terminal. WCDMA capacity is approximately double that of narrowband CDMA. The larger bandwidth and the use of both coherent demodulation and fast power control in the uplinks and the downlinks allow a lower receiver threshold. WCDMA uses a network protocol signalling similar to that of GSM therefore, it will be able to use the existing GSM network as the core network infrastructure.

In addition to the improvements of data throughput and interworking, 3G will provide an additional spectrum for the operators. The increase in $3 \mathrm{G}$ spectrum efficiency will also provide the operator with more throughputs over limited resources. The transition from the existing $2 \mathrm{G}$ networks to $3 \mathrm{G}$ capabilities will evolve over time. Seamless handover and roaming capabilities are provided by the attempt by dual-mode terminals [12].

$3 \mathrm{G}$ has evolved from $2 \mathrm{G}$ and is built on the success of GSM (GSM, GSM1800, and GSM1900). Dual-mode terminals ease migration from $2 \mathrm{G}$ to $3 \mathrm{G}$. Although many options for $3 \mathrm{G}$ exist, the radio technology in $3 \mathrm{G}$ will likely be Wideband CDMA (Collision detect multiple access).

\section{Fourth Generation}

While $3 \mathrm{G}$ wireless networks are still on the developing stage, researchers are working on $4 \mathrm{G}$ cellular networks with cellular data rates of $20 \mathrm{Mbps}$ and beyond. The high data rate of $4 \mathrm{G}$ cellular phones leads the users to watch high-resolution movies and television programs on their cellular phones.

$4 \mathrm{G}$ referred to IMT-Advanced [19] (International Mobile Telecommunications Advanced), as defined by ITU-R. It's based on an all-IP packet switched network. In $4 \mathrm{G}$ Peak data rates of up to approximately $100 \mathrm{Mbit} / \mathrm{s}$ for high mobility such as mobile access and up to approximately $1 \mathrm{Gbit} / \mathrm{s}$ for low mobility such as nomadic/local wireless access. It dynamically share and use the network resources to support more simultaneous users per cell and channel bandwidth $5 \mathrm{MHz}$ to $20 \mathrm{MHz}$ and optionally up to $40 \mathrm{MHz}$.

The Peak link spectral efficiency of $4 \mathrm{G}$ is $15 \mathrm{bit} / \mathrm{s} / \mathrm{Hz}$ in the downlink, and $6.75 \mathrm{bit} / \mathrm{s} / \mathrm{Hz}$ in the uplink. System spectral efficiency of up to $3 \mathrm{bit} / \mathrm{s} / \mathrm{Hz} / \mathrm{cell}$ in the downlink and 2.25 $\mathrm{bit} / \mathrm{s} / \mathrm{Hz} / \mathrm{cell}$ for indoor usage.

\section{Fifth Generation}

Fifth generation mobile internet can be completely wireless communication without limitation it brings us perfect real world wireless World Wide Wireless Web (WWWW). It supports by LAS-CDMA, OFDM, MC-CDMA, UWB, IPV6 hence it is said to be real wireless world of mobile internet networks.

The 5G technology mobile communication visions are:

1. Smart device explosion from 5 Billion to 5 Trillion 
devices.

2. Thrusts to derive new business models with super-fast hyper connection.

3. Provides new solution for cost effective system.

4. Giga service demands by display technology and Giga networks such as AR, Hologram and UHD.

5. Nationwide knowledge based service for all people.

Fig 2 shows the $5 \mathrm{G}$ architecture. It has the separate Data plane and Control plane. Table 2 shows the 5G technology requirements. The $5 \mathrm{G}$ technology has intends to resolve several service trends, due to the improvement in the smart phones technology multimedia service require more and more bandwidth. Since the cloud service has the enormous development it extends its hands on mobile too with rapid development. Augmented reality and Virtual reality are said to be immersive multimedia technology which provides digitally enhanced view of the real world which can able to connect us with more meaningful contents in our everyday life. These technology mainly focus on the camera and sensors in a smartphone or tablets which adds layers of digital information such as videos, photos and sounds directly on top of items around us. Social networking websites also expands their view with rich multimedia contents. Machine to Machine communication devices are rapidly spreading with requirement of high data rate media based services. Personalized services such as Location based services, $\mathrm{u}$-health are integrated into humans daily life.

Introduction of smartphone terminals will continue to rise worldwide traffic share. In 2012 the traffic share raised by $44.2 \%$ by 2017 it raises to $67.5 \%$. Smartphones generate tremendous traffic when compared to feature phones, smartphones acquire 24 times and tablet acquires 122 times of the feature phone traffic. Due to the improvement in the device environment such as high resolution, real 3D display and holographic view acquire more bandwidth. Global Exponential mobile data traffic increases with the growth rate of approximately $2 \mathrm{x}$ per year to $1000 \mathrm{x}$ in a decade.

The $5 \mathrm{G}$ mobile environment comprises of:

1. User friendly system.

2. Highly reliable and secure.

3. Can acquire thousands of time of mobile devices.

4. Can able to handle thousands of times of mobile traffic.

5. Reduction in latency to milliseconds with real time response.

6. Low battery consumption.

7. Cloud system based services.

8. Realistic applier services.

9. Personalized services.

10. Ultra-fast and high traffic data.

Threats in $5 \mathrm{G}$ implementations are specified below:

1. The 5G implementation needs share a common core network infrastructure by all the service providers, since there is possibilities of compromising by a single operator to collapse the whole network infrastructure.

2. Fraudulent in billing and theft of service by third parties may occurs.
3. Since $5 \mathrm{G}$ is purely based on IP, it will be vulnerable to all the available security issues in the current web world.

4. Spoofing, eavesdropping and phishing attacks may be familiar in this infrastructure.

Table 2. 5 G Requirements.

\begin{tabular}{ll}
\hline Parameters & Specifications \\
\hline Data rates & $1-10 \mathrm{Gbps} / 100 \mathrm{~s}$ of Mbps \\
Capacity & $36 \mathrm{~TB} / 500 \mathrm{~GB} /$ month/user \\
Spectrum & Higher frequencies \& flexibility \\
Energy & $\sim 10 \%$ of today's consumption \\
Latency reduction & $\sim 1 \mathrm{~ms}$ \\
Reliability & $99.999 \%$ \\
Coverage & $>20 \mathrm{~dB}$ of LTE \\
Battery & $\sim 10$ years \\
\hline
\end{tabular}

\section{Conclusion}

Cellular telephone has evolved in many ways. The transmission is now become digital, and new bands of frequencies have been allocated. Video conferencing have been developed. All of these advances still rely on the cellular concept of reusing frequencies in multiple small cells within a contiguous service area to achieve the necessary capacity within a limited spectrum.

\section{References}

[1] Advanced Mobile Phone System, Bell System Technical Journal, January, 1979.

[2] Bates, R. Wireless Broadband Handbook. McGraw Hill, 2001.

[3] Bekkers, R. and Smits, J. Mobile Telecommunications. Artech, 2000 .

[4] Gibson, J., et al. The Communications Handbook. CRC Press, 1997.

[5] Gralla, P. How Wireless Works. Que, 2001.

[6] Guizzo, E. "Closing in on the Perfect Code." IEEE Spectrum, March 2004, pp. 36-42.

[7] Jagoe, A. Mobile Location Services: The Definitive Guide. Prentice Hall, 2002.

[8] Dropman, Ulrich, "A real step toward UMTS," http://w2.siemens.de/telcom/articles/e0497/497drop.htm.

[9] $3 \mathrm{G}-$ The Future of Communications, http://www.gsmworld.com/technology/3g_future.htm.

[10] Gorham, Peter, "Strategic Technology Steps on the CDMAONE Evolution Path to 3G CDMA2000," 3G Mobile Broadband Conference, August 10, 1999.

[11] Mercer Management Consulting, "3G Investment: How will it Prove in?," $3 \mathrm{G}$ Mobile Broadband Conference, August 10, 1999.

[12] Buckingham, Simon, "High Speed Circuit-Switched Data (HSCSD), http://www.mobileipworld.com/wp/ffz_wp3.htm.

[13] "An Overview of GPRS, http://www.gsmworld.com/technology/gprs.html. 
[14] ITU Telecommunications indicators update-2009. www.itu.int/ITU-D/ict/statistics/

[15] 3G Americas. List of $3 \mathrm{G}$ deployments worldwide. www.3gamericas.org

[16] UMTS Forum. www.umts-forum.org

[17] Holma, H. et al. "High-Speed Packet Access Evolution in 3GPP Release 7." IEEE Communications Magazine, 45(12):29-35, December 2007.

[18] Holma, H. and A. Toskala. "High-Speed Downlink Packet Access." Chapter 11. WCDMA for UMTS. New York: John Wiley \& Sons, Inc., 2002.
[19] 3GPP specification: Requirements for further advancements for E-UTRA (LTE Advanced)

[20] 3GPP Technical Report: Feasibility study for Further Advancements for E-UTRA (LTE Advanced)

[21] “5G WIRELESS ARCHITECTURE-2010” By Vadan Mehta.

[22] Amos Edward Joel (Bell Labs), "Cellular Mobile Communication System.” 